\title{
AN AUTOMATED APPROACH TO STEREO MATCHING SEASAT IMAGERY
}

\author{
Mia Denos \\ Department of Photogrammetry and Surveying \\ University College London \\ Gower Street \\ London WC1E 6BT
}

\begin{abstract}
This paper describes a procedure developed at University College London for the automatic stereo matching of SAR imagery from NASA's Seasat satellite. The method employed uses Gruen's least squares correlation technique to improve the match accuracy of randomly generated points as they are cascaded down an image pyramid, coupled with a sheet growing mechanism in order to produce a dense array of points. The overall result of the cascading procedure is the totally automatic production of a dense digital disparity model [DDM].
\end{abstract}

\section{INTRODUCTION}

In order to apply the area based Otto-Chau [5] stereo matcher to any form of imagery, you must first accurately determine image conjugate points. These points then become seedpoint data for the matcher and act as starting points for its sheet growing mechanism. Without accurately determined image conjugate points the stereo matching process is unable to proceed.

The Otto-Chau stereo matcher was developed under the aegis of the Alvey MMI-137 [Real-time 2.5D Vision System] project undertaken at University College London. Otto-Chau is an area based stereo matcher which incorporates both Gruen's [3] adaptive least squares technique and a sheet growing mechanism.

To date, any seedpoint data needed for the Otto-Chau algorithm has been measured manually via an interactive display program on a personal workstation. However, this procedure is both time consuming and prone to error [1].

In an attempt to overcome these problems and to achieve a totally automated system, cascade [6] was developed. Cascading uses the idea that if stereo images are reduced in size by a large enough factor, then any randomly generated points can be transformed into accurate conjugate points in the original images by applying Gruen's least squares correlation technique at each tier of the image pyramid (fig. 1). Gruen's [3] least squares correlation technique is used to act as a "pull in" to improve the match accuracy at the new increased resolution which is associated with each consecutive tier of the image pyramid. And, by also applying the sheet growing mechanism of the Otto-Chau [5] stereo matcher near the top of the image pyramid it is possible to produce a dense stereo matched extent at the bottom of the image pyramid.

It must be noted that if applying the technique of cascading to SAR imagery, before any stereo matching can take place the problem of speckle $[1,2]$ must be addressed. Speckle is perceived by the Otto-Chau matcher to be a series of micro disparity domains, the boundaries of which act to inhibit the growth of the texture dependent sheet growing mechanism. This means that if a conventional approach to stereo matching is made, the matched extent will be limited to the immediate area surrounding the seedpoints [1]. However, it should also be noted that the technique of cascading points down an image pyramid means that the prohibitive effect of speckle in the SAR imagery will be not encountered until, at the earliest, the bottom two tiers of the image pyramid. To overcome the problem of speckle the images must be convolved with an appropriate speckle reduction filter [4].

\section{METHODOLOGY}

The first stage in the overall process is to convolve the left and right images of the stereo pair with a speckle reduction filter. For this series of experiments an adaptive 
median filter was used to achieve the goal. The reason for convolving the stereo images with a speckle reduction filter has been previously discussed in the introduction.

The first stage of the actual cascading process involves the of reduction the stereo images by pixel grey level averaging. The reduction factor employed needs to be specified by the operator at the outset, and it determines the number of tiers in the image pyramid.

The second stage of the cascading process involves the use of a random number generator to generate conjugate points in the reduced images. These points are then cascaded through the image pyramid, whilst using Gruen's least squares technique at each new tier in order to increase match accuracy at the new improved resolution.

In order to achieve a well dispersed, dense array of stereo matched points which extend over the stereo area of the bottom tier, the sheet growing mechanism of the Otto-Chau stereo matcher needs to be employed at a stage when the randomly generated points have been cascaded through several tiers in the image pyramid.

The refining and sheet growing procedures are then repeated until the bottom tier (ie. the original, speckle reduced imagery) has been successfully stereo matched.

\section{RESULTS}

The data set selected was SEASAT [7] images of Death Valley, California, USA (map ref. $36^{\circ} 19^{\prime} \mathrm{N} 116^{\circ} 52^{\prime} \mathrm{W}$ ). The SEASAT images used were; left; rev. 882 file number 08820946, and right; rev. 1140 file number 11400008 . Stereo sub-images of $1024 \times 1024$ pixels were extracted for the experimentation (figs $2 \mathrm{a}$ and $2 \mathrm{~b}$ ).

Table 1 gives details of applying the cascading automatic stereo matching technique to the SEASAT stereo images. Two cases are presented; the first with no speckle reduction having been applied to the images before processing (fig $2 \mathrm{c}$ ); the second case being when the images were first convolved with an adaptive median filter prior to processing (fig $2 \mathrm{~d}$ ).

\section{DISCUSSION}

Table 1 shows that there is a need for the use of a speckle reduction. In the no speckle reduction case, the cascading technique works well until the bottom two tiers of the image pyramid, then the percentage coverage obtained goes down from $68.6 \%$ to $58.5 \%$. In the case where a speckle reduction filter was applied to the images beforehand, the final extent of the stereo matched coverage was $81 \%$. It should be noted that the percentage coverage can never be as high as 100 simply because the percentage overlap is not that high, as can be seen from figs $2 a$ an $2 b$. Figs $2 c$ and $2 d$ represent the stereo matched extent pictorially.

From looking at figs $2 \mathrm{c}$ and $2 \mathrm{~d}$, it is quite noticeable that the basin shown in the Death Valley imagery has failed to match successfully. This is a very positive result for the cascading process. The SEASAT images were recorded a different times and because the floor of the basin is a salt lake, the time differential will mean that the basin floor may have experienced some degree of physical change. This means that the cascading process only works in stereo areas, and is not prone to erroneous matches if this sort of artifact exists in the imagery.

\section{CONCLUSION}

The technique of cascading in order to automatically stereo match SEASAT imagery has been described. The method has been shown to be able to yield a dense DDM, without the need of any operator intervention in a reasonably short time period, typically less than 6 CPU hours on a SUN4.

The technique of cascading [6], whether to stereo match imagery or to locate conjugate points, is a very powerful imaging processing tool. Not only because it is automatic, fast and requires no prior knowledge of the sensor geometry, but because its application could extend far beyond the stereo matching of SEASAT imagery as demonstrated here. Any imagery which contains a large number of discontinuities, occlusion and shadows [6], may well to benefit from the application of this technique. 


\section{ACKNOWLEDGEMENTS}

I would like to thank Mark O'Neill from the Department of Photogrammetry and Surveying, University College London and IBM Scientific Centre, Winchester, for all their support. Also, NASA for supplying the SEASAT imagery.

\section{REFERENCES}

[1] Denos Mia, A pyramidal scheme for stereo matching SIR-B imagery, Lets. Int. J. of Remote Sensing, submitted, 1991.

[2] Goodman Joseph W., A random walk through the field of speckle, Optical Engineering, 25(5): pp 610-612, May 1986.

[3] Gruen A.W., Adaptive least squares correlation, a powerful image matching technique, S. Afr. J. of Phot., Remote Sensing and Cart., 14(3): pp175-187, 1985.

[4] Lee Jong-See, Speckle reduction and analysis for synthetic aperture radar images, Optical Engineering, 25(5): pp 636-643, May 1986.

[5] Otto G.P. and Chau T.K.W., A region growing algorithm for the matching of terrain images, Image and Vision Computing, 7(2): pp 83-93, 1989.

[6] O'Neill M.A. and Mia Denos, A practical approach to the stereo matching of urban imagery, Image and Vision Computing [accepted for publication], 1991.

[7] Pravado S.H., B. Hunneycutt, B.M. Holt and D.N. Held, Seasat synthetic aperture radar data user's manual, JPL Publication 82-90, March 1983.

\begin{tabular}{|c|c|c|c|c|c|c|}
\hline & & No Spec & Reduction & Speckle & eduction & \multirow{11}{*}{$\begin{array}{l}\text { Point at } \\
\text { which sheet } \\
\text { growing } \\
\text { commences } \\
-\quad-\quad-\end{array}$} \\
\hline $\begin{array}{c}\text { Tier } \\
\text { Number }\end{array}$ & $\begin{array}{l}\text { Image } \\
\text { Size }\end{array}$ & $\begin{array}{l}\text { Points } \\
\text { Matched }\end{array}$ & $\begin{array}{c}\text { Percentage } \\
\text { Coverage }\end{array}$ & $\begin{array}{c}\text { Points } \\
\text { Matched }\end{array}$ & $\begin{array}{c}\text { Percentage } \\
\text { Coverage }\end{array}$ & \\
\hline 1 & $4 \times 4$ & 3000 & N/A & 3497 & N/A & \\
\hline 2 & $8 \times 8$ & 1053 & N/A & 1866 & N/A & \\
\hline 3 & $16 \times 16$ & 247 & N/A & 453 & N/A & \\
\hline 4 & $32 \times 32$ & 392 & $38.5 \%$ & 312 & $30.5 \%$ & \\
\hline 5 & $64 \times 64$ & 2160 & $52.7 \%$ & 2198 & $53.7 \%$ & \\
\hline 6 & $128 \times 128$ & 10911 & $66.6 \%$ & 9655 & $58.9 \%$ & \\
\hline 7 & $256 \times 256$ & 44974 & $68.8 \%$ & 46353 & $70.7 \%$ & \\
\hline 8 & $512 \times 512$ & 161893 & $61.8 \%$ & 205020 & $78.2 \%$ & \\
\hline $9^{*}$ & $1024 \times 1024$ & 153222 & $58.5 \%$ & 212330 & $81.0 \%$ & \\
\hline
\end{tabular}

Table 1. Table Showing Cascading Results

N/A = Not Applicable

$*=2$ pixel grid used

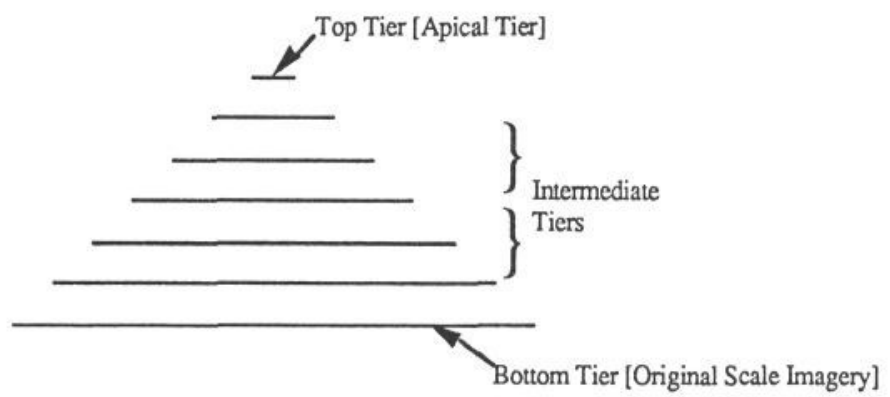

Figure 1. Schematic of an Image Pyramid 


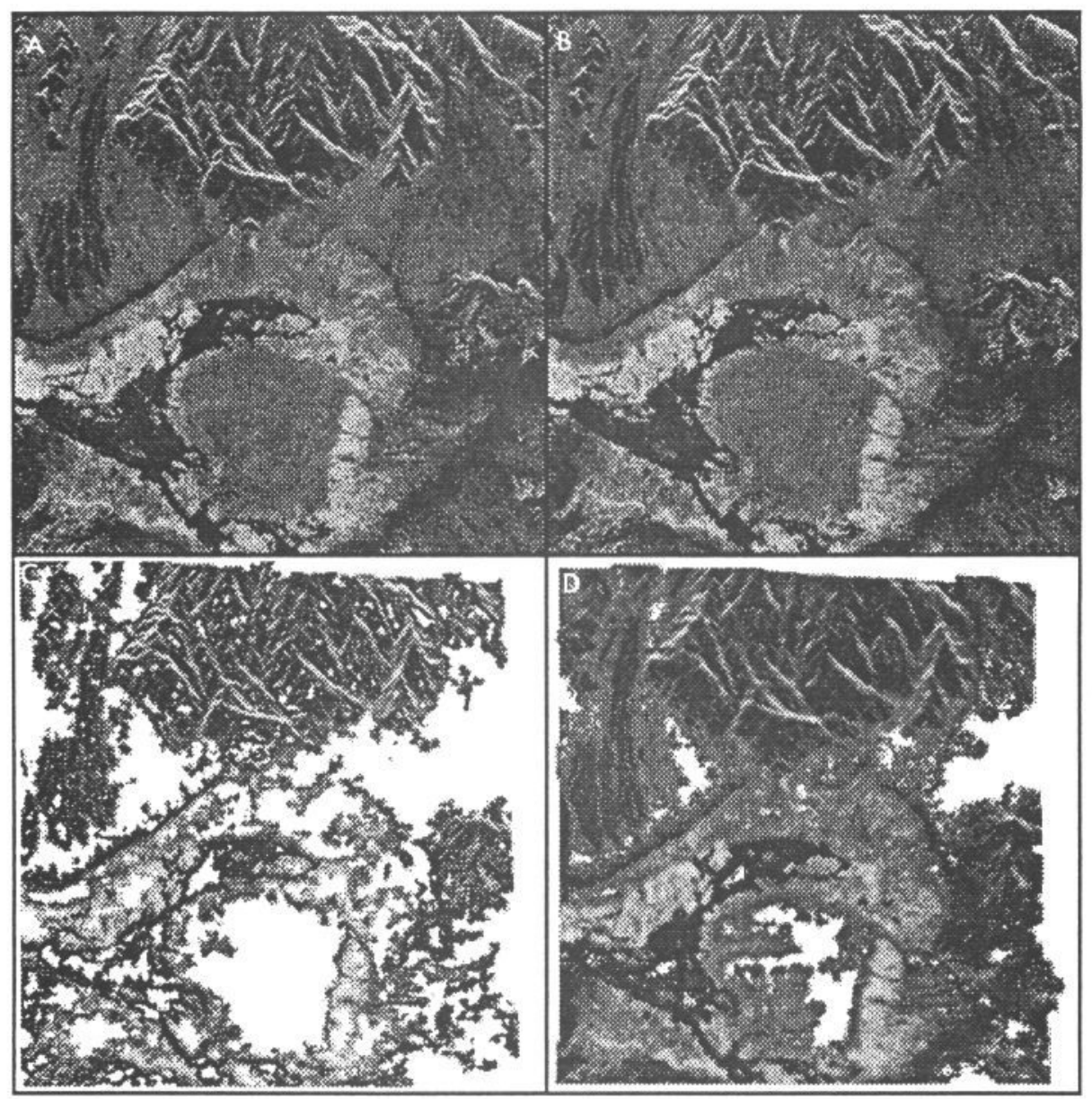

Eigure 2 .

a) Left SEASAT Image of Death Valley.

b) Right SEASAT Image of Death Valley.

c) Stereo Matched Extent in Non Speckle Reduction Case.

d) Stereo Matched Extent in Speckle Reduction Case. 